\title{
CORSEN, a new software dedicated to microscope-based 3D distance measurements: mRNA-mitochondria distance, from single-cell to population analyses
}

\author{
LAURENT JOURDREN, ${ }^{1,2}$ THIERRY DELAVEAU, ${ }^{1,3}$ EMELIE MARQUENET, ${ }^{1,3}$ CLAUDE JACQ, ${ }^{1,3}$ \\ and MATHILDE GARCIA ${ }^{1,3}$ \\ ${ }^{1}$ Institute of Biology of the École Normale Supérieure, CNRS UMR8197, INSERM U1024, 75230 Paris Cedex 05, France \\ ${ }^{2}$ Plate-forme Transcriptome, 75230 Paris Cedex 05, France \\ ${ }^{3}$ Functionnal Genomics Section, Laboratoire de Génétique Moléculaire, 75230 Paris Cedex 05, France
}

\begin{abstract}
Recent improvements in microscopy technology allow detection of single molecules of RNA, but tools for large-scale automatic analyses of particle distributions are lacking. An increasing number of imaging studies emphasize the importance of mRNA localization in the definition of cell territory or the biogenesis of cell compartments. CORSEN is a new tool dedicated to three-dimensional (3D) distance measurements from imaging experiments especially developed to access the minimal distance between RNA molecules and cellular compartment markers. CORSEN includes a 3D segmentation algorithm allowing the extraction and the characterization of the cellular objects to be processed-surface determination, aggregate decomposition-for minimal distance calculations. CORSEN's main contribution lies in exploratory statistical analysis, cell population characterization, and high-throughput assays that are made possible by the implementation of a batch process analysis. We highlighted CORSEN's utility for the study of relative positions of mRNA molecules and mitochondria: CORSEN clearly discriminates mRNA localized to the vicinity of mitochondria from those that are translated on free cytoplasmic polysomes. Moreover, it quantifies the cell-to-cell variations of mRNA localization and emphasizes the necessity for statistical approaches. This method can be extended to assess the evolution of the distance between specific mRNAs and other cellular structures in different cellular contexts. CORSEN was designed for the biologist community with the concern to provide an easy-to-use and highly flexible tool that can be applied for diverse distance quantification issues.
\end{abstract}

Keywords: 3D imaging; FISH; distance measurements; population analysis

\section{INTRODUCTION}

Forty-five years ago, the discovery of the mRNA molecule (Jacob and Monod 1964) was the first step in explaining how a molecule can sense the geometrical structure of the cell. In fact, genomic technologies applied to metazoan organisms have shown that the life cycle of an mRNA molecule is a highly regulated process from transcription to translation and degradation at different specific cellular loci (McKee and Silver 2007). It is usually accepted that transcript localization is a mechanism allowing the sequestration of mRNAs mainly in the cellular region in which the encoded protein is re-

Reprint requests to: Mathilde Garcia, Institute of Biology of the École Normale Supérieure, CNRS UMR8197, INSERM U1024, 46 Rue d'Ulm, 75230 Paris Cedex 05, France; e-mail: mathilde.garcia@ens.fr; fax: 331-4432-3941; or Claude Jacq, Institute of Biology of the École Normale Supérieure, CNRS UMR8197, INSERM U1024, 46 Rue d'Ulm, 75230 Paris Cedex 05, France; e-mail: claude.jaqc@ens.fr; fax: 33-1-4432-3941.

Article published online ahead of print. Article and publication date are at http://www.rnajournal.org/cgi/doi/10.1261/rna.1996810. quired (Lecuyer et al. 2007). Nevertheless, it is important to keep in mind that mRNA is an elusive molecule whose localization and stability are highly flexible. This is why rigorous quantitative approaches are required to describe transcript dynamics accurately. Significant progress has been made in visualizing single particles of mRNA (Long et al. 1995; Chartrand et al. 2000), but tools to analyze the data in terms of intracellular distances remain scarce.

In this work we developed a new program, CORSEN, to carry out systematic distance analyses in the study of mRNA localization in the vicinity of mitochondria. Indeed, microarray analyses in yeast Saccharomyces cerevisiae revealed the existence of more than 500 mitochondria-localized mRNAs (MLRs) that are encoded in the nuclear genome but translated to the close proximity of mitochondria (Marc et al. 2002; Saint-Georges et al. 2008). Their corresponding protein is likely to be further imported in the organelle via a cotranslational process. The large number of existing MLRs brings to light the importance of mRNA asymmetric localization for 
mitochondrial biogenesis. Furthermore, recent genomewide analyses discriminated at least two MLR classes according to the nature of the mRNA-binding proteins that ensure the mitochondrial localization (Saint-Georges et al. 2008).

Pioneer studies that aimed to validate these data using a microscopy approach (Garcia et al. 2007a) pointed out several experimental difficulties that justify the development of a quantitative three-dimensional (3D) distance analysis tool: (1) Since mitochondria occupy a large part of the cell volume, deciding whether an mRNA particle is significantly close to the mitochondrion surface requires rigorous statistical analyses; (2) The mitochondria morphology is highly flexible and growth and cell dependent; and (3) Cell-to-cell variations according to mRNA-organelle distances are substantial in isogenic growth cultures. If this cell heterogeneity can be explained by different biological parameters (asynchronous growth conditions, petite formation), it has to be seriously analyzed to draw reliable conclusions.

CORSEN is an original tool devoted to the specific issue of 3D distance analysis. Indeed, despite the recent vertiginous advances in microscopy techniques (Rodriguez et al. 2007; Kherlopian et al. 2008), image analysis remains a main bottleneck in extracting relevant data from cell imaging experiments (Carpenter 2007). Commercial microscopy packages (Metamorph, ImagePro) that provide important functionalities are available, but they lack flexibility. Furthermore, no combination of available software can fulfill the properties of our program. Some valuable open-source projects emerged during the past few years, including ImageJ (http://rsbweb. nih.gov/ij/) (Collins 2007), a Java-based package for general image analysis, or other tools like CellProfiler (Carpenter et al. 2006) and CelliD (Gordon et al. 2007) that were specially designed for cell automated analysis. Nevertheless, none of them are fully implemented for 3D analysis and distance measurements. Here we report an efficient algorithm that quantifies mRNA to mitochondria distances and analyzes the corresponding cell distribution. CORSEN is the first full package designed for biologists to address cellular distance quantification issues. Moreover, CORSEN's main contribution relies on its ability to perform population analyses. Semiautomated digital image acquisition coupled with automated image analysis can be conducted on a large cell population. This quantitative methodology can reveal subpopulation features that are masked in population averages and cannot be detected by human eye analyses.

\section{RESULTS}

\section{Overview of CORSEN software}

An overview of CORSEN functions is presented in Figure 1 and briefly described below:

1. 3D segmentation and extraction of object features (inner coordinates, surface coordinates, center of mass, to- tal intensity, mean intensity, median intensity, maximal intensity, volume, density, sphericity). This first step was implemented as a plug-in for the ImageJ platform. A simple threshold-based segmentation method is applied. An iterative inclusion-test algorithm is then performed to associate objects found in the different planes that share common areas. In order to remain flexible, this segmentation step was not coupled to any image processing. Therefore, users remain free to perform their own refinement prior to this stage. For instance, in the work presented below, the image processing procedure we developed allowed an automatic extraction of both small particles and aggregates, which could not be performed using a unique threshold (this method is also available as a macro for ImageJ on the CORSEN website; see Materials and Methods).

2. Distance assessment was performed using an independent Java-based program. Depending on the morphology of the objects to be compared, different calculation options have to be considered. Indeed, minimal distance between mass centers can be used to analyze the relative positions of small objects within the cell, like single molecules of mRNA. However, it is not accurate enough to analyze distances between the mitochondrial tubular network and mRNAs that could form aggregates in its vicinity. For mitochondria, the surface's voxels were considered for distance calculations. To solve the problem of mRNA aggregates whose mass center could not be representative of the position of the included mRNAs, we implemented an algorithm that performs object decomposition. During this step, a 3D cuboid grid is placed on the objects. For each cuboid, the mass center is computed according to the intensity of the included object and used to calculate the distance to the closest mitochondria surface. Distances are intensity weighted and set negative if cuboid mass centers are included in mitochondria. CORSEN is then able to generate a histogram of the cellular intensityweighted distance distribution. Other distance calculation methods are available that answer diverse biological concerns (see the CORSEN documentation).

3. The CORSEN application includes a visualization interface. A 3D reconstitution of the analyzed cell can be generated based on a marching cube algorithm. Calculated distances can be visualized together with cellular objects. Users can apply filters on intensity, distance, or size to visualize particles of special interest.

4. Population studies can be easily performed since CORSEN was implemented for automatic analyses of a cellular series. Quantitative data from the calculated distance distribution are extracted for each cell, depending on the user settings (median, maximal, or minimal distance; percentage of particles under a given distance threshold), and a histogram corresponding to the whole population is generated. Individual and population distribution tables can be saved for further statistical analyses, for example, with R software. 


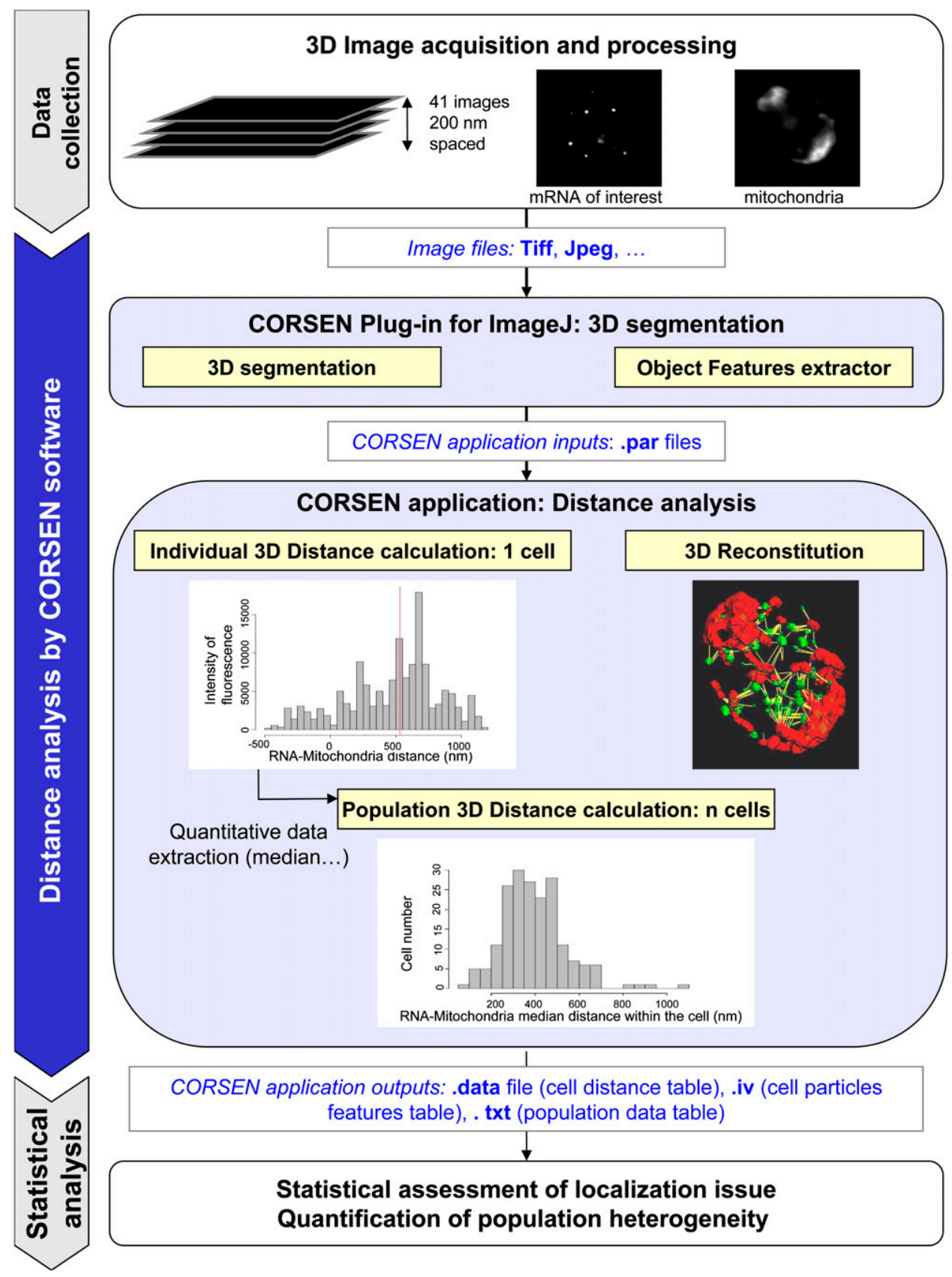

FIGURE 1. CORSEN overview: from cell imaging to quantitative assessment of mRNA localization. The pipeline of an analysis of 3D distances with CORSEN software is presented (see the text for more detail). Format needed at each step is indicated in blue. CORSEN analysis proceeds in two steps: (1) CORSEN plug-in for ImageJ performs a 3D segmentation and object feature extractions: this step generates a text file (.par) summarizing all the characteristics of the objects to be analyzed. (2) CORSEN application allows distance analyses between two objects of interest (here, mRNA and mitochondria). For each cell, distances are calculated and can be visualized in the 3D reconstitution module (mitochondria, red; mRNA, green; calculated distances, yellow lines). CORSEN can be used for automatic analysis of cellular series; in this case, quantitative data from cell distance distribution (for instance, median distance) are extracted and distribution within the population is plotted. Tables summarizing cell distance (.data), cell features (.iv), and population analysis (.txt) are saved and can be used for further statistical analyses.

\section{CORSEN availability and usability}

The CORSEN application is an open-source software package (GNU public license version 2 or later, CeCILL-A) available for the biologist community. The 3D segmentation algorithm is designed as a plug-in for the commonly used ImageJ plat- form. The distance application is an independent Java-based program allowing distance calculations, basic statistical analyses, and 3D reconstitution of analyzed cells. Both versions are available at http://transcriptome.ens.fr/corsen.

CORSEN has been designed with the constant concern of providing an easy-to-use and highly flexible tool that 
addresses quantitatively intracellular localization issues. To install and launch the CORSEN application, a simple click on the CORSEN website is needed. The website includes detailed documentation including demonstration images to help new users familiarize themselves with every step of the analysis. The source code is written in Java in order to be compatible with diverse computer systems (Linux, Windows including Vista, and Mac OS X [still experimental]). CORSEN analyses can be conducted on the various gray image formats that are handled by Image $\mathrm{J}$ ( 8 or 16 bit). CORSEN output tables that summarize cell features, cell distances, and population distances are suited for further statistical analyses with Microsoft Excel or R software. The graphical user interface allows easy settings of CORSEN's options and analyses for nonprogrammers. CORSEN can address different biological localization issues thanks to several distance calculation options according to the shape of the objects to be analyzed. Moreover, the source code is available at the Google code forge (http:// code.google.com/p/corsen), so that programmers can add functions to expand CORSEN functionalities and report bugs. Our implementation handles the actual multicore generation of processors, which improves the calculation speed: this allows it to run computationally intensive tasks needed for cell series analyses. The duration of analyses varies according to the image size, the number of objects found per cell, and the chosen distance calculation option, but distance calculation only requires several seconds per cell on standard desktop computers.

\section{DISCUSSION}

\section{CORSEN allows a quantitative and statistical assessment of mRNA localization}

To demonstrate CORSEN's utility, we first addressed the question of the dissimilarity between the two well-characterized mRNA populations, MLR and non-MLR. These populations were defined by comparison of mRNA associated with mitochondrial fractions to the whole cell mRNA population, using microarray or real-time quantitative polymerase chain reaction analyses (Garcia et al. 2007a; Saint-Georges et al. 2008). Taking advantage of data from mitochondrial-encoded mRNA, the results were normalized to calculate mRNA percentages of association with mitochondria. Final results were corrected in order to take into account mitochondrial fraction contaminations by cytosol. This allowed us to discriminate between the mRNAs translated in the vicinity of mitochondria (localization percentage $>8 \%$ ) and the ones that were translated by free polysomes in the cytosol (localization percentage $\leq 8 \%$ ). Fluorescence in situ hybridization (FISH) experiments were performed on five mRNAs: TRX2, which is not related to mitochondrial biogenesis (localization $=0 \%$ ); three MLR, ATP2 (localization = 45\%), ATP3 (localization = $88 \%$ ), and BCS1 (localization $=31 \%$ ); and one non-MLR, ATP16 $($ localization $=0 \%)($ Saint-Georges et al. 2008). The results of our analysis conducted with CORSEN are presented in Figure 2.

When applied to TRX2 mRNA (Fig. 2A), CORSEN allowed us to define a reference population (147 cells) whose distribution of median mRNA distance to mitochondria had a mean value of $420 \mathrm{~nm}$, which is compatible with the yeast cytoplasm dimensions. This reference distribution was compared with the ones obtained with the three MLR, ATP2, $A T P 3$, and BCS1, and the non-MLR, ATP16 (Fig. 2B-E). The obtained results were completely coherent with our previous biochemical results (Fig. 2F). The result for ATP16 $($ mean $=380 \mathrm{~nm} / P$-value $=0.22)$ corresponds to the expected symmetrical distribution. In contrast, the distribution of median distance between ATP2, ATP3, and BCS1 mRNAs and the mitochondrial surface unambiguously corresponds to an asymmetrical position in the yeast cytoplasm: mean values were $130 \mathrm{~nm}(P$-value $<2.2 \mathrm{e}-16), 220 \mathrm{~nm}(P$-value $<$ $2.2 \mathrm{e}-16)$, and $255 \mathrm{~nm}(P$-value $=5.9 \mathrm{e}-10)$, respectively. In conclusion, our unbiased analysis clearly distinguishes the mRNAs localized in the vicinity of mitochondria from those that are translated on free cytoplasmic polysomes.

\section{CORSEN allows quantification of cell population heterogeneity}

Our reference population allowed us to define a median distance threshold (160 nm) (Fig. 2A-E, black lines) under which mRNAs are considered to be asymmetrically localized within the cell. This 5\% error threshold was used to assess the mRNA localization cell by cell.

In this way, CORSEN software can help to compare the mRNA localization between two different genetic strains of $S$. cerevisiae. For instance, the two laboratory wild-type strains, Gi252 and BY4743 (a derivative of S288c), could be distinguished by CORSEN analyses (Fig. 3). Whereas ATP2 mRNA asymmetric localization was $70 \%$ in the Gi252 strain, this value, although still significant, was only $20 \%$ in the BY4743 strain. This difference might be connected to the fact that the Gi252 strain has a reduced respiratory capacity, probably due to the insertion of a defective Ty1 retrotransposon in the HAP1 coding sequence (Gaisne et al. 1999).

Quite surprisingly, CORSEN analyses on ATP2, ATP3, and BCS1 mRNA in the Gi252 strain revealed that 23\%, $60 \%$, and $46 \%$, respectively, of the cell population does not exhibit an mRNA asymmetric distribution. Indeed, for each MLR analyzed with CORSEN, we detected two distinct cell populations: (1) cells with a clear association between mRNA and the mitochondrial surface, and (2) cells showing no asymmetrical distribution of mRNA.

This emphasizes the superiority of the quantitative FISHCORSEN analyses over global biochemical analyses, which can only offer mean distance values. Also, this may indicate that, in batch cultures, the mitochondria biogenesis of yeast cells is not synchronized as it was shown in the model of the yeast metabolic cycle ( $\mathrm{Tu}$ et al. 2005). 


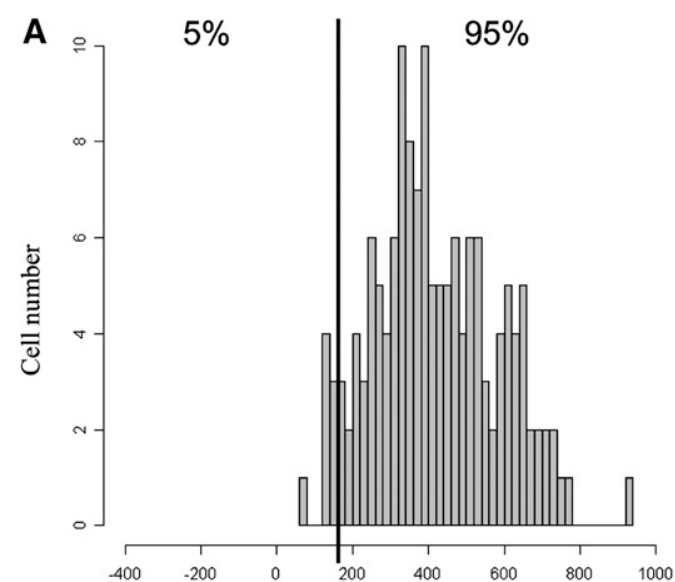

RNA-Mitochondria median distance within the cell (nm)

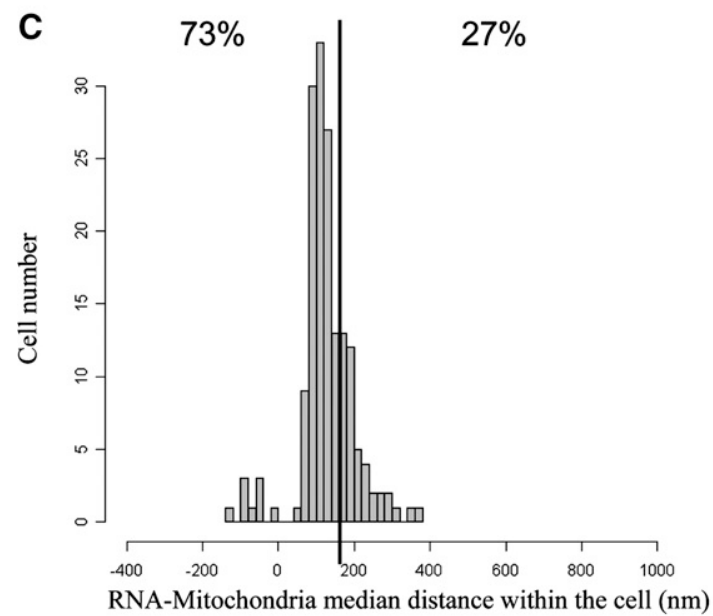

E

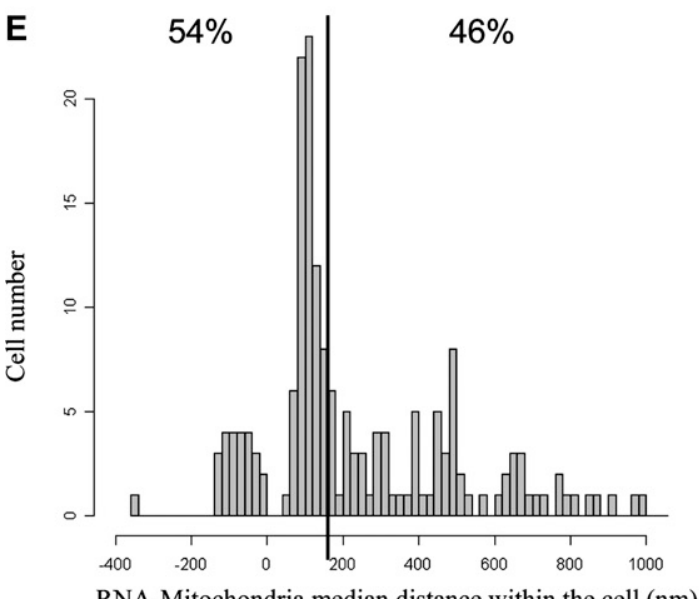

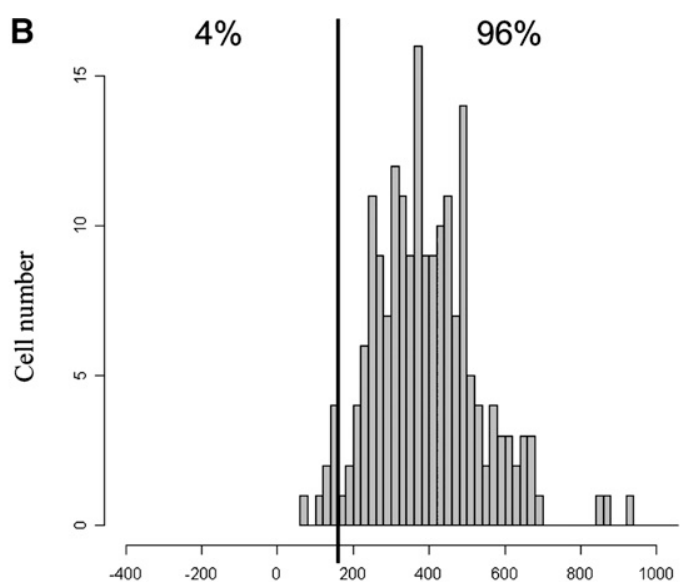

RNA-Mitochondria median distance within the cell (nm)

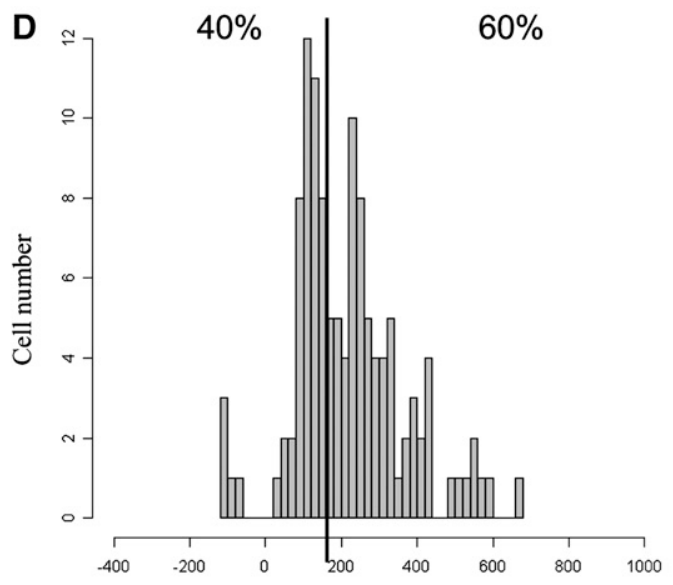

RNA-Mitochondria median distance within the cell $(\mathrm{nm})$



FIGURE 2. Validation of CORSEN for assessment of mRNA localization to mitochondria. CORSEN was used to determine the distribution of mRNA to mitochondria median distance in the Saccharomyces cerevisiae Gi252 strain for five mRNA: (A) TRX2 (147 cells analyzed), (B) ATP16 (190 cells analyzed), (C) ATP2 (165 cells analyzed), (D) ATP3 (119 cells analyzed), and (E) BCS1 (179 cells analyzed). The vertical bar (A-E) indicates the median distance threshold $(160 \mathrm{~nm})$, under which mRNA is considered to be asymmetrically localized within the considered cell. This corresponds to $5 \%$ of cells in the negative control $(A)$, and, for each distribution, the percentages of cells under and above the 160-nm threshold are indicated. $(F)$ A box-plot representation of the distributions is shown; the bottom and top of the box correspond to the 25th and 75th percentile (the lower and upper quartiles, respectively), and the band near the middle of the box corresponds to the 50th percentile (the median). 


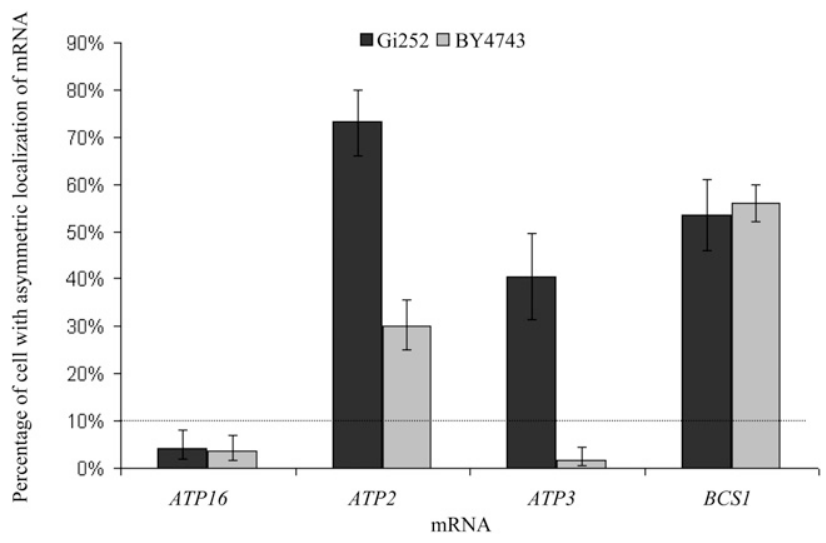

FIGURE 3. Quantification of variation of population heterogeneity with CORSEN. Comparison of the percentage of cells with asymmetric localization of ATP16, ATP2, ATP3, and ATP16 in two different Saccharomyces cerevisiae strains, GI252 and BY4743. These percentages were calculated using the $160-\mathrm{nm}$ threshold according to the TRX2 reference population distribution. For each measure, a confident interval is calculated based on a binomial distribution. The dotted line indicates the threshold above which the mRNA is assumed to be asymmetrically localized with an expected false-positive rate of $<5 \%$.

An increasing number of studies demonstrate that mRNA abundances are only a weak surrogate for the protein concentrations, and that mRNA localizations are much better indicators of actual gene expression activities (Mata et al. 2005). CORSEN will enable biologists to carry out large-scale quantitative analyses of the distance between fluorescent RNA probes and other cellular compartment markers.

\section{MATERIAL AND METHODS}

\section{FISH experiments}

We used the S. cerevisiae diploid strains Gi252 (a derivative of W303) and BY4743 (a derivative of S288c). After growth over one night in YPGal medium (2\% galactose, $1 \%$ bactopeptone, $1 \%$ yeast extract), cells in the mid-log exponential phase (OD $=0.3-0.6)$ were collected for FISH experiments following our previously described protocol for multitarget detection (Garcia et al. 2007b). Mitochondria were detected using probes against ribosomal RNA, together with probes against the mRNA that we wanted to localize. 3D imaging was performed after hybridization, by acquiring 41 images, $200 \mathrm{~nm}$ apart, along the $z$-axis on a Olympus IX81 upright microscope (Olympus) using a $100 \times \mathrm{NA}=1.4$ objective.

Supplemental Table S1 summarizes the different probes used in this study. The aminoallyl-modified thymidines are in bold type.

\section{Image processing}

We developed an image processing procedure using a unique threshold for automatic extraction of both small particles and aggregates that could not be segmented together (this method is available as a macro for ImageJ on the CORSEN website). The procedure is based on the generation of two distinct images, one containing the tiny objects, the other containing the brightest ones, that are then added up to retrieve all of the significant signals. After applying a threshold on the minimal gray value to remove the cellular background, the tiny object image $\left(\mathrm{I}_{1}\right)$ is obtained by performing a Laplacian filter $(r=2)$ that enhances small particle signals, followed by a median filter ( $r=2$ for mitochondria; $r=1$ for mRNA). The brightest particle image $\left(\mathrm{I}_{2}\right)$ is obtained simply by applying a high threshold on the minimal gray value to the initial image. Selection of the two thresholds needed for generation of $\mathrm{I}_{1}$ and $\mathrm{I}_{2}$ is automated using an analysis of the cell intensity histogram. According to object occupancies, the background threshold values are chosen as follows: $T=m+c \cdot \sigma$ with $T$ being the threshold gray value; $m$, the cellular median intensity; $\sigma$, the cellular standard deviation of intensity; and c, a coefficient determined by object occupancy. Typically, for $\mathrm{I}_{1}$ generation, we set $c=-2$ for mitochondria objects and $c \in[-1,1]$ for RNA objects depending on their expression level. For $\mathrm{I}_{2}$ generation, the $\mathrm{c}$ value was raised by 2 units. Adding up the $\mathrm{I}_{1}$ and $\mathrm{I}_{2}$ results in an intensity-enhanced image that can be submitted to isodata automatic threshold. After eroding (median filter with $\mathrm{r}=2$ for mitochondria and $r=1$ for $\mathrm{mRNA}$ ), this binary image was used as a mask on the initial image to set all background pixels to zero, whereas selected object pixels kept their initial intensity. This final image was analyzed using the CORSEN 3D segmentation algorithm with the minimal threshold value set to 1 .

\section{SUPPLEMENTAL MATERIAL}

Supplemental material can be found at http://www.rnajournal.org.

\section{ACKNOWLEDGMENTS}

We thank Xavier Darzacq, Stephane Le Crom, Yann Saint-Georges, and Frederic Devaux for precious advice and comments. We are very grateful to Thomas Portnoy and Baptiste Saudemont for their careful reading of the manuscript. This work was supported by grants from the Agence Nationale de la Recherche (ANR) (BLAN0234-01) and the Association pour la Recherche sur le Cancer (ARC) $($ A08/2/1034).

Received November 13, 2009; accepted April 13, 2010.

\section{REFERENCES}

Carpenter AE. 2007. Software opens the door to quantitative imaging. Nat Methods 4: 120-121.

Carpenter AE, Jones TR, Lamprecht MR, Clarke C, Kang IH, Friman O, Guertin DA, Chang JH, Lindquist RA, Moffat J, et al. 2006. CellProfiler: Image analysis software for identifying and quantifying cell phenotypes. Genome Biol 7: R100.

Chartrand P, Bertrand E, Singer RH, Long RM. 2000. Sensitive and high-resolution detection of RNA in situ. Methods Enzymol 318: 493-506.

Collins TJ. 2007. ImageJ for microscopy. Biotechniques 43: 25-30.

Gaisne M, Becam AM, Verdiere J, Herbert CJ. 1999. A 'natural' mutation in Saccharomyces cerevisiae strains derived from S288c affects the complex regulatory gene HAP1 (CYP1). Curr Genet 36: 195-200.

Garcia M, Darzacq X, Delaveau T, Jourdren L, Singer RH, Jacq C. 2007a. Mitochondria-associated yeast mRNAs and the biogenesis of molecular complexes. Mol Biol Cell 18: 362-368.

Garcia M, Darzacq X, Devaux F, Singer RH, Jacq C. 2007b. Yeast mitochondrial transcriptomics. Methods Mol Biol 372: 505-528. 
Gordon A, Colman-Lerner A, Chin TE, Benjamin KR, Yu RC, Brent R. 2007. Single-cell quantification of molecules and rates using open-source microscope-based cytometry. Nat Methods 4: 175181.

Jacob F, Monod J. 1964. Biochemical and genetic mechanisms of regulation in the bacterial cell. Bull Soc Chim Biol (Paris) 46: 14991532.

Kherlopian AR, Song T, Duan Q, Neimark MA, Po MJ, Gohagan JK, Laine AF. 2008. A review of imaging techniques for systems biology. BMC Syst Biol 2: 74.

Lecuyer E, Yoshida H, Parthasarathy N, Alm C, Babak T, Cerovina T, Hughes TR, Tomancak P, Krause HM. 2007. Global analysis of mRNA localization reveals a prominent role in organizing cellular architecture and function. Cell 131: 174-187.

Long RM, Elliott DJ, Stutz F, Rosbash M, Singer RH. 1995. Spatial consequences of defective processing of specific yeast mRNAs revealed by fluorescent in situ hybridization. RNA 1: $1071-1078$.
Marc P, Margeot A, Devaux F, Blugeon C, Corral-Debrinski M, Jacq C. 2002. Genome-wide analysis of mRNAs targeted to yeast mitochondria. EMBO Rep 3: 159-164.

Mata J, Marguerat S, Bahler J. 2005. Post-transcriptional control of gene expression: A genome-wide perspective. Trends Biochem Sci 30: $506-514$.

McKee AE, Silver PA. 2007. Systems perspectives on mRNA processing. Cell Res 17: 581-590.

Rodriguez AJ, Condeelis J, Singer RH, Dictenberg JB. 2007. Imaging mRNA movement from transcription sites to translation sites. Semin Cell Dev Biol 18: 202-208.

Saint-Georges Y, Garcia M, Delaveau T, Jourdren L, Le Crom S, Lemoine S, Tanty V, Devaux F, Jacq C. 2008. Yeast mitochondrial biogenesis: A role for the PUF RNA-binding protein Puf3p in mRNA localization. PLoS One 3: e2293.

Tu BP, Kudlicki A, Rowicka M, McKnight SL. 2005. Logic of the yeast metabolic cycle: Temporal compartmentalization of cellular processes. Science 310: 1152-1158. 

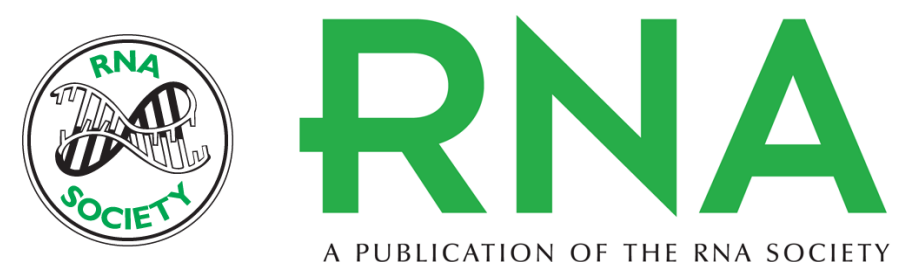

A PUBLICATION OF THE RNA SOCIETY

\section{CORSEN, a new software dedicated to microscope-based 3D distance measurements: mRNA-mitochondria distance, from single-cell to population analyses}

Laurent Jourdren, Thierry Delaveau, Emelie Marquenet, et al.

RNA 2010 16: 1301-1307 originally published online May 21, 2010

Access the most recent version at doi:10.1261/rna.1996810

Supplemental Material

References

License

Email Alerting Service
http://rnajournal.cshlp.org/content/suppl/2010/05/17/rna.1996810.DC1

This article cites 18 articles, 4 of which can be accessed free at: http://rnajournal.cshlp.org/content/16/7/1301.full.html\#ref-list-1

Receive free email alerts when new articles cite this article - sign up in the box at the top right corner of the article or click here. 